\title{
Tratamiento con desmopresina sublingual en dos lactantes con hidranencefalia y diabetes insípida central
} Treatment with sublingual desmopressin in two infants with hydranencephaly and central diabetes insipidus

\author{
Dr. Gustavo R. Marin ${ }^{a}$, Dra. Berta Baspineiro ${ }^{b}$ y Dra. Iris Vilca
}

\begin{abstract}
RESUMEN
La diabetes insípida central es una patología infrecuente en pediatría ocasionada por un déficit de vasopresina. Sus manifestaciones clínicas principales son poliuria y polidipsia. Las malformaciones cerebrales son una de las principales causas. La desmopresina es la droga sintética de elección para el tratamiento. Una de las vías de administración es la sublingual y su uso en lactantes es muy limitado. Se describe a dos lactantes con hidranencefalia y diabetes insípida central que fueron tratados satisfactoriamente con desmopresina sublingual. Palabras clave: diabetes insípida central, desmopresina, hidranencefalia, malformaciones del sistema nervioso.
\end{abstract}

\section{ABSTRACT \\ Central diabetes insipidus is a rare disease in children caused by a deficiency of vasopressin. Its main clinical manifestations are polyuria and polydipsia. Brain malformations are one of the main causes. Desmopressin is the synthetic drug of choice for the treatment. One of the routes of administration is sublingual and its use in infants is very limited. We describe two infants with central diabetes insipidus and hydranencephaly who were successfully treated with sublingual desmopressin. \\ Keywords:centraldiabetesinsipidus, desmopressin, hydranencephaly, nervous system malformations.}

http: / / dx.doi.org/10.5546/ aap.2018.e93

Cómo citar: Marín GR, Baspineiro B, Vilca I. Tratamiento con desmopresina sublingual en dos lactantes con hidranencefalia y diabetes insípida central. Arch Argent Pediatr 2018;116(1):e93-e97.

a. Unidad de Nefrología, Hospital Materno Infantil "Héctor Quintana", Jujuy, Argentina.

b. Servicio de Clínica Pediátrica, Hospital Materno Infantil "Héctor Quintana", Jujuy, Argentina.

Correspondencia:

Dr. Gustavo R. Marín: viltipocovalefer@gmail.com

Financiamiento: Ninguno.

Conflicto de intereses: Ninguno que declarar.

Recibido: 5-3-2017

Aceptado: 7-7-2017

\section{INTRODUCCIÓN}

La diabetes insípida central (DIC) es una patología que se manifiesta con poliuria y polidipsia debido a un déficit de la hormona antidiurética vasopresina. La incidencia se estima entre 3 y 4 casos por 100000 habitantes. ${ }^{1}$

Las malformaciones cerebrales representan una de las principales causas ${ }^{2-7} \mathrm{y}$, entre ellas, la hidranencefalia, caracterizada por ausencia de gran parte del tejido supratentorial, puede asociarse a una DIC. ${ }^{4}$

La desmopresina, una droga análoga sintética de la vasopresina, es el fármaco de elección para tratar la DIC. Se puede administrar por vía oral, nasal, parenteral y sublingual. ${ }^{1,3,5,8-10}$ La desmopresina sublingual (DS) es la última formulación liberada al mercado y el uso en lactantes con DIC es muy limitado, pero, al parecer, con buenos resultados. ${ }^{10-13}$

El objetivo de esta comunicación es describir el uso de la DS en dos lactantes con DIC e hidranencefalia, con resultados satisfactorios.

\section{Caso 1}

Lactante varón que nació de embarazo a término, sin antecedentes patológicos, con microcefalia y peso de 2580 gramos.

La ecografía cerebral y la resonancia magnética evidenciaron hidranencefalia (Figura 1.A-B). Sin otras alteraciones clínicas destacables, al momento del egreso, se planificó el seguimiento con un equipo multidisciplinario.

A los 4 meses, se internó por síndrome bronquiolítico, con un peso entre el percentilo $20 \mathrm{y}$ 50, normohidratado, con leve retraso madurativo. $\mathrm{Su}$ alimentación era lactancia materna exclusiva. Durante esta internación, presentó registros elevados de diuresis hipotónica que le ocasionaron hipernatremia intermitente corregida con hidratación oral. Recibió broncodilatadores y corticoides, con respuesta satisfactoria; al egresar, continuó en seguimiento multidisciplinario.

Dos meses después, reingresó con signos de obstrucción bronquial y deshidratación 
moderada. El análisis de laboratorio relevante mostró natremia: $167 \mathrm{mmol} / \mathrm{L}$; potasemia: 5,7 mmol/L; uremia: $106 \mathrm{mg} / \mathrm{dl}$; creatininemia: $1,06 \mathrm{mg} / \mathrm{dl}$; acidosis metabólica y calcio iónico de $0,97 \mathrm{mmol} / \mathrm{L}$. Recibió broncodilatadores, corticoides, corrección con gluconato de calcio e hidratación oral; 12 horas después, la natremia bajó a $150 \mathrm{mmol} / \mathrm{L}$ y se registró una diuresis de $3,9 \mathrm{cc} / \mathrm{kg} /$ hora.

Ante la reiteración del cuadro bronquial, se evaluó un posible reflujo gastroesofágico y aspiración, y se detectó reflujo sin trastorno aspirativo.
Posteriormente, se registraron natremias que oscilaron entre 152 y $168 \mathrm{mmol} / \mathrm{L} \mathrm{y}$ diuresis entre 3,5 y 7,8 cc/ $\mathrm{kg} /$ hora. Se hidrató con dificultad y presentó varios picos febriles sin foco infeccioso. Un análisis de laboratorio urinario evidenció sodio: $98 \mathrm{mmol} / \mathrm{L}$; potasio: $5 \mathrm{mmol} / \mathrm{L}$; cloro: $35 \mathrm{mmol} / \mathrm{L}$ (con natremia de $152 \mathrm{mmol} / \mathrm{L}$ ). Se sospechó DIC por la asociación de malformación cerebral, hipertonicidad plasmática, hipotonicidad urinaria y poliuria.

$\mathrm{Al}$ reducirse la carga renal de solutos de la leche (manteniendo un aporte calórico adecuado), la poliuria bajó parcialmente, pero la natremia fue oscilante (147-167 mmol/L).

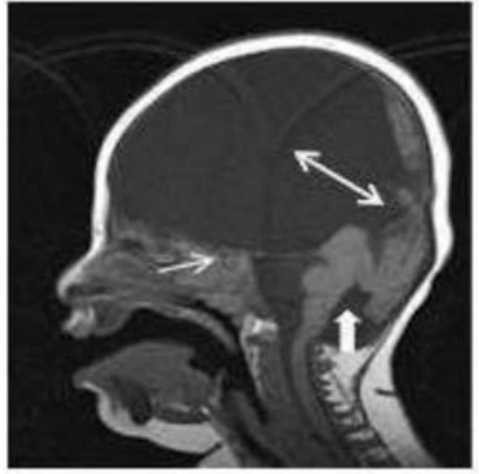

A

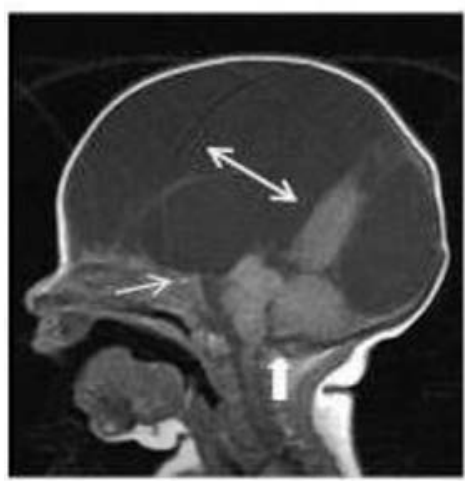

C

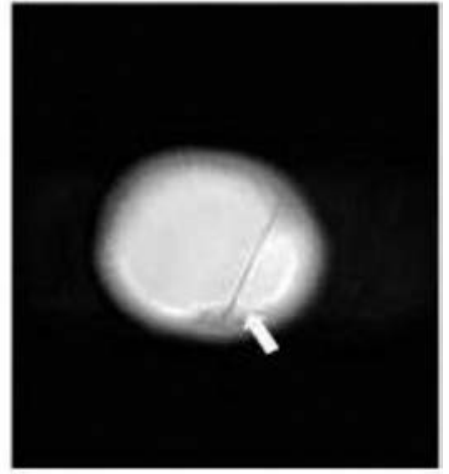

B

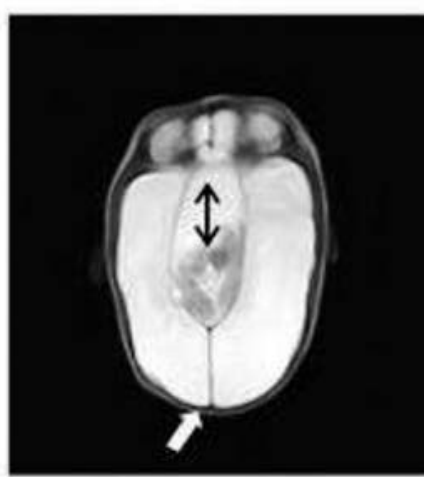

D

Caso 1. A: Imagen sagital T1. Región supratentorial sin masa cerebral, llena de líquido cefalorraquídeo, remanentes de lóbulos parietooccipitales $(\longleftrightarrow$ ), región infratentorial con preservado tronco cerebral, atrofia cerebelosa ( $\Rightarrow$ ) y posible pituitaria hipoplásica $(\longrightarrow)$

B: Imagen axial T2. Líquido cefalorraquídeo sin masa cerebral, falx cerebri desviado $(\Rightarrow)$

Caso 2. C: Imagen sagital T1. Región supratentorial sin masa cerebral, llena de líquido cefalorraquídeo, remanente de lóbulo temporal $(\longleftrightarrow)$, región infratentorial con tronco cerebral y cerebelo presentes $(\vec{b})$, posible pituitaria hipoplásica $(\longrightarrow$ ) D: Imagen axial T2. Líquido cefalorraquídeo, tercer ventrículo dilatado, tálamo malformado $(\longleftrightarrow)$, falx cerebri posterior preservado $(\Rightarrow)$ 
Se administraron 24 ug de DS. El análisis de laboratorio predesmopresina mostró natremia: $155 \mathrm{mmol} / \mathrm{L}$; osmolaridad plasmática: $318 \mathrm{mOsmol} / \mathrm{kg}$; osmolaridad urinaria: $183 \mathrm{mOsmol} / \mathrm{kg}$; 24 horas después, natremia: $138 \mathrm{mmol} / \mathrm{L}$; osmolaridad plasmática: $286 \mathrm{mOsmol} / \mathrm{kg}$; urinaria: $540 \mathrm{mOsmol} / \mathrm{kg}$. La diuresis bajó de 4,4 a 1,9cc/ kg/hora (Tabla 1). Estos datos confirmaron el diagnóstico de DIC. Se detectó hipocortisolemia matinal con dosaje de hormonas tiroideas normales y se suplió con corticoides.

Continuó con 12 ug de desmopresina cada 12 horas. El control de la natremia y la diuresis fue satisfactorio. Al momento del egreso, pesó 7600 gramos, con natremia de $146 \mathrm{mmol} / \mathrm{L}$ y diuresis de $2,2 \mathrm{cc} / \mathrm{kg} /$ hora, y se alimentaba con leche maternizada al $15 \%$. El seguimiento se planificó con el equipo multidisciplinario.

Durante tres meses de seguimiento, la natremia se mantuvo estable $(140-144 \mathrm{mmol} / \mathrm{L})$. Registró un pico de $149 \mathrm{mmol} / \mathrm{L}$, que se normalizó aumentando el aporte hídrico. El progreso ponderal fue adecuado. Se mantiene la dosis de desmopresina en 12 ug sublingual cada 12 horas.

\section{Caso 2}

Lactante femenino que nació de un embarazo a término sin complicaciones. Se internó por síndrome de aspiración meconial de leve a moderado, que requirió oxigenoterapia nasal. El Apgar al nacer fue de 8/9; pesó 3150 gramos y se detectó microcefalia. La ecografía y la resonancia magnética cerebral revelaron hidranencefalia (Figura 1. C-D). La evolución respiratoria fue favorable y egresó en 12 días con un peso de 3680 gramos.
A los 2 meses de vida, reingresó con una neumonía lobar derecha y deshidratación de moderada a grave. Recibió antibioticoterapia de amplio espectro, expansión con solución fisiológica a $60 \mathrm{cc} / \mathrm{kg} /$ dosis e hidratación oral. Recuperó un $7 \%$ del peso. La diuresis fue de $4,6 \mathrm{cc} / \mathrm{kg} / \mathrm{hora}$, con natremia de $162 \mathrm{mmol} / \mathrm{L}$, con sodio-potasio urinarios de 89 y $22 \mathrm{mmol} / \mathrm{L}$, respectivamente.

Reinició la alimentación con leche maternizada al $15 \%$ por sonda nasogástrica debido a la succión defectuosa. El cuadro infeccioso evolucionó favorablemente, pero persistieron registros de hipernatremia y poliuria con lento progreso ponderal.

Presumiendo DIC, se le administraron 12 ug de DS cada $12 \mathrm{~h}$. El análisis de laboratorio predesmopresina mostró natremia: $152 \mathrm{mmol} / \mathrm{L}$; osmolaridad plasmática: $313 \mathrm{mOsmol} / \mathrm{kg}$; osmolaridad urinaria: $197 \mathrm{mOsmol} / \mathrm{kg}$; 24 horas después, natremia: $144 \mathrm{mmol} / \mathrm{L}$; osmolaridad plasmática: $296 \mathrm{mOsmol} / \mathrm{kg}$; urinaria: $320 \mathrm{mOsmol} / \mathrm{kg}$. La diuresis bajó de $5,5 \mathrm{cc} / \mathrm{kg} /$ hora a $2,3 \mathrm{cc} / \mathrm{kg} /$ hora y se confirmó el diagnóstico (Tabla 1). Se comprobó hipocortisolemia matinal y hormonas tiroideas normales, y se suplió con corticoides.

Continuó con 12 ug de DS cada 12 horas y leche maternizada al 15\%. A la semana, se rotó a desmopresina oral por falta de disponibilidad de sublingual. Al mes de internada, egresó al domicilio con alimentación enteral continua con sonda nasogástrica, un peso de 4125 gramos, diuresis de $4 \mathrm{cc} / \mathrm{kg} /$ hora y natremia de $144 \mathrm{mmol} / \mathrm{L}$. Quedó en control multidisciplinario y resta evaluar su trastorno deglutorio.

En la Tabla 1, se observa la evolución.

TABLA 1. Respuesta clínica y de laboratorio a la desmopresina sublingual

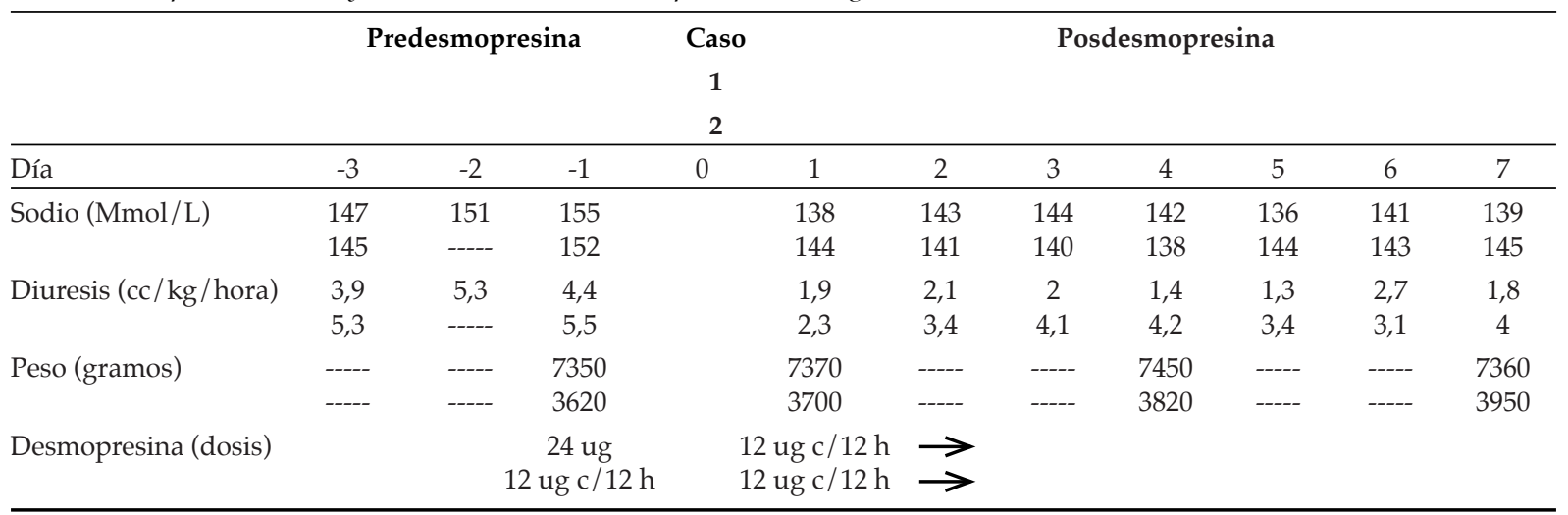




\section{DISCUSIÓN}

El tratamiento de una DIC en lactantes es complicado. Por un lado, se utiliza la desmopresina para reducir la poliuria ${ }^{1,8} \mathrm{y}$, por otro, un lactante requiere un elevado aporte lácteo para alcanzar un desarrollo normal. En este escenario, si la hidratación no se planifica correctamente, existe el riesgo de retención hídrica e hiponatremia, la complicación más frecuente de la desmopresina.

Para disminuir la poliuria, se ha recurrido, con relativo éxito, a la disminución de la carga renal de solutos de la leche y uso de diuréticos tiazídicos al inicio o ante dificultades con el uso de desmopresina. Sin embargo, estos tratamientos pueden ocasionar trastornos hidroelectrolíticos y repercusiones en el crecimiento por modificaciones en las fórmulas lácteas. ${ }^{3,14-15}$

La vía oral (preferentemente) y la nasal son las más utilizadas para el tratamiento de una DIC; la DS se ha comenzado a utilizar recientemente ${ }^{1,6-13}$

Episodios hiponatrémicos frecuentes, dificultades en la dosificación, técnica y patologías locales dificultan el uso nasal. ${ }^{1,3,-7,715} \mathrm{La}$ administración oral, aunque menos habitual, no está exenta de complicaciones hiponatrémicas, ${ }^{5-8}$ sobre todo, cuando hay malformaciones cerebrales. ${ }^{6}$

De acuerdo con la experiencia clínica, pareciera que la absorción de la DS por la mucosa bucal logra controles de natremia y diuresis más regulares que por otras vías., ${ }^{3,9-12} \mathrm{Al}$ tener mayor biodisponibilidad que los comprimidos, se emplean dosis más bajas. Se recomienda iniciar con 2-5 ug/ kg/ día cada 12 horas $^{1,8,11}$ y regularla de acuerdo con la natremia y la diuresis, aunque, en relación con la dosis, puede haber una gran variabilidad individual en la respuesta terapéutica.

Cinco neonatos y 3 lactantes (6 con malformaciones cerebrales de la línea media) que recibieron DS en una dosis media de $3 \mathrm{ug} / \mathrm{kg} / \mathrm{d}$ normalizaron la natremia y la diuresis en alrededor de 72 horas sin registrar hiponatremia, incluso, en seguimiento a largo plazo. ${ }^{9-12}$

En 15 lactantes con DIC y malformaciones cerebrales, se modificó la solución nasal para el uso en la mucosa bucal. La natremia se mantuvo estable durante el seguimiento de once meses. ${ }^{3}$

En otro estudio, la DS se administró a 14 lactantes con DIC, y ningún paciente requirió internación por disnatremia en un período de vigilancia de 5 años. ${ }^{13}$

Sobre la base de la experiencia previa, ${ }^{10,11}$ en nuestro caso, se diluyó un comprimido de 120 microgramos de DS en 5 cc de agua destilada $(1 \mathrm{cc}=24 \mathrm{ug})$. Esta solución se refrigeró hasta 5 días para mantener la estabilidad de la droga. ${ }^{1,14}$ En ambos pacientes normohidratados, se administraron $0,5 \mathrm{cc}(12 \mathrm{ug})$ cada 12 horas (3-6 ug $/ \mathrm{kg} / \mathrm{d}$ ) y se reguló el aporte de líquidos diarios sobre la base de la diuresis (suma de los pesos de cada pañal) para evitar una sobrecarga hídrica. Este esquema logró mantener la eunatremia y la diuresis estable, incluso, en uno de ellos, tres meses después, sin registros de hiponatremia y desarrollo pondoestatural normal.

En conclusión, aunque la experiencia clínica es escasa, la DS es una alternativa terapéutica útil para tratar lactantes con DIC sin requerir diuréticos y modificaciones nutricionales. La preparación y administración de la droga resultó sencilla para los padres, lo que restó complejidad al tratamiento. Para evitar complicaciones, se requiere un manejo correcto de la hidratación, sobre todo, en situaciones clínicas que la comprometan (ej.: fiebre, gastroenteritis), y un contacto estrecho con un equipo de especialistas encargado del seguimiento de estos pacientes.

\section{REFERENCIAS}

1. Di Iorgi N, Morana G, Napoli F, et al. Management of diabetes insipidus and adipsia in the child. Best Pract Res Clin Endocrinol Metab 2015;29(3):415-36.

2. Werny D, Elfers C, Perez FA, et al. Pediatric central diabetes insipidus: Brain malformations are common and few patients have idiopathic disease. J Clin Endocrinol Metab 2015;100(8):3074-80.

3. Smego AR, Backeljauw P, Gutmark-Little I. Buccally administered intranasal desmopressin acetate for the treatment of neurogenic diabetes insipidus in infancy. $J$ Clin Endocrinol Metab 2016;101(5):2084-8.

4. Santiprabhob J, LikitmaskulS, Boonyasiri A, et al. Etiologies of central diabetes insipidus in thai children. J Pediatr Endocrinol Metab 2005;18(7):653-61.

5. Ooi HL, Maguire AM, Ambler GR. Desmopressin administration in children with central diabetes insipidus: a retrospective review. J Pediatr Endocrinol Metab 2013;26(1112):1047-52.

6. Rizzo V, Albanese A, Stanhope R. Morbidity and mortality associated with vasopressin replacement therapy in children. J Pediatr Endocrinol Metab 2001;14(7):861-7.

7. Karthikeyan A, Abid N, Sundaram P, et al. Clinical characteristics and management of cranial diabetes insipidus in infants. J Pediatr Endocrinol Metab 2013;26(1112):1041-6.

8. Oiso Y, Robertson GL, Nørgaard JP, et al. Clinical review: Treatment of neurohypophyseal diabetes insipidus. J Clin Endocrinol Metab 2013;98(10):3958-67.

9. Kappy MS, Sonderer E. Sublingual administration of desmopressin effectiveness in an infant with holoprosencephaly and central diabetes insipidus. Am J Dis Child 1987;141(1):84-5.

10. DeWaeleK,Cools M, DeGuchtenaere A, et al.Desmopressin lyophilisate for the treatment of central diabetes insipidus: 
first experience in very young infants. Int JEndocrinol Metab 2014;12(4):e16120.

11. Korkmaz HA, Demir K, Kılıç FK, et al. Management of central diabetes insipidus with oral desmopressin lyophilisate in infants. J Pediatr Endocrinol Metab 2014;27(910):923-7.

12. Hanta D, Törer B, Temiz F, et al. Idiopathic central diabetes insipidus presenting in a very low birth weight infant successfully managed with lyophilized sublingual desmopressin. Turk J Pediatr 2015;57(1):90-3.

13. Juul KV, Schroeder M, Rittig S, et al. National surveillance of central diabetes insipidus (CDI) in Denmark: Results from 5 years registration of 9309 prescriptions of desmopressin to 1285 CDI patients. JClin Endocrinol Metab 2014;99(6):2181-7.

14. Rivkees SA, Dunbar N, Wilson TA. The management of central diabetes insipidus in infancy: desmopressin, low renal solute load formula, thiazide diuretics. J Pediatr Endocrinol Metab 2007;20(4):459-69.

15. Abraham MB, Rao S, Price G, et al. Efficacy of hydrochlorothiazide and low renal solute feed in neonatal central diabetes insipidus with transition to oral desmopressin in early infancy. Int J Pediatr Endocrinol 2014;2014(1):11. 